\title{
Assessment of the tolerability, and appetite suppressing effect, of a midmorning snack containing high molecular weight polysaccharides, which form a gel in the stomach
}

\author{
E. J. Simpson ${ }^{1}$, E. Woods ${ }^{1}$, S. Gaunt ${ }^{2}$, D. Park ${ }^{2}$, T. I. Foster ${ }^{3}$ and A. Macdonald ${ }^{1}$ \\ ${ }^{1}$ School of Life Sciences University of Nottingham NG72UH, ${ }^{2}$ Eminate Limited, University of Nottingham, LE12 5RD \\ and ${ }^{3}$ School of Biosciences University of Nottingham LE12 5RD
}

The increased prevalence of overweight individuals in the western population has coincided with an increase in the consumption of energy dense snacks between meals and the frequency of snack food consumption ${ }^{(1)}$. Snacking may contribute to excess dietary energy intake (EI) if compensation in the amount of food eaten at subsequent meals does not occur ${ }^{(2)}$. However, a differential in the satiating properties of macronutrients has been demonstrated, with protein or poorly digested carbohydrate consumption resulting in reduced intake at the next meal ${ }^{(3)}$, such that snacks rich in these macronutrients could play a role in reducing daily EI.

This study aimed to assess the gastrointestinal (GI) tolerability and appetite suppressant properties of a snack containing a combination of xanthan/א-carrageenan and galactomannan/glucomannan, using a double-blinded, placebo-controlled, randomised, cross-over design.

Six healthy, normal weight men (23(5.3)yrs, $\left.22.4(1.7) \mathrm{kg} / \mathrm{m}^{2}\right)$, initially completed a 3 day diary for characterisation of GI habits. They then attended the laboratory for 2 study visits, separated by a week, and taking place in the morning after fasting from midnight the night before. A standard (low-fibre) meal (5.8 MJ) was consumed on the evening before each visit. On arrival, subjects completed an appetite assessment (Combined Appetite Score; CAS), and reported any adverse GI symptoms experienced in the previous 24 hrs. At $t=0 \mathrm{~min}$, they were given a low-fibre cereal breakfast $(705 \mathrm{~kJ})$ and at $150 \mathrm{~min}$, subjects were given either the test or control snack to consume. Ninety minutes later, an ad libitum pasta meal was provided. Subjects completed the CAS at intervals throughout the day and GI symptom diaries were maintained for $48 \mathrm{hrs}$ following each study visit. Figures display the mean with SEM.

Fasting appetite was similar on both visits $(P=0.564)$, and each eating episode resulted in a significant fall in the CAS (Fig. 1$)$. There were no statistical differences in the CAS between the two study days $(P=0.270)$, or in the 90 min following snack consumption $(P=0.486)$. Despite the appetite rating being similar prior to consumption of the ad libitum meal, 5 of the 6 subjects consumed at least $630 \mathrm{~kJ}$ less (mean 1.9 MJ, range 0.6-3.8 MJ) on the test visit, resulting in the group mean being significantly lower on this visit when compared to the control day (Fig. 2; $P<0.05$ ). The sixth subject consumed a similar amount on both study visits $(3.23 \mathrm{MJ}$ vs. $3.26 \mathrm{MJ}$ ). No subject reported any change in bowel habits (frequency or change in Bristol Stool scale), or GI discomfort during or following either study visit.

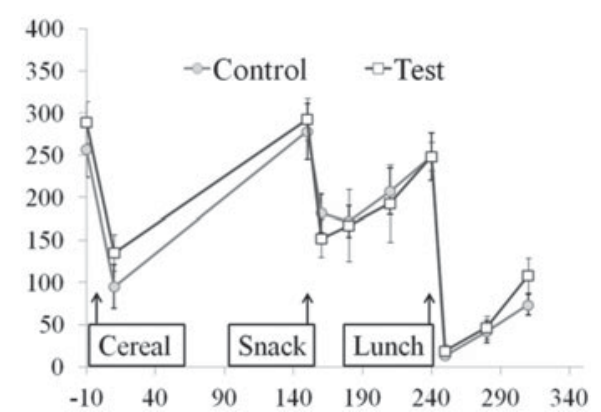

Fig. 1. Combined Appetite Score.

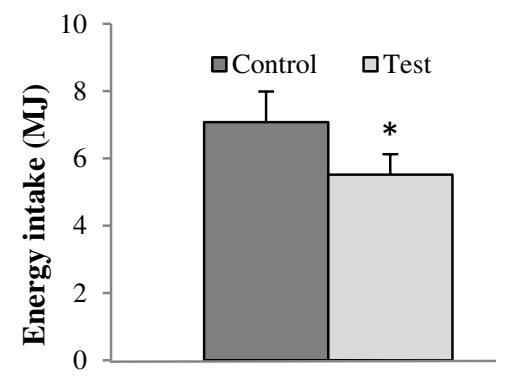

Fig. 2. Energy intake at the ad libitum meal.

Despite subjective appetite being unaffected, consuming a mid-morning snack containing a mix of high molecular weight polysaccharides, reduced energy intake at the next meal.

1. Zizza C, Siega-Riz AM, Popkin BM (2001) Significant increase in young adults' snacking between 1977-1978 and $1994-1996$ represents a cause for concern! Prev Med Apr; 32(4), 303-10.

2. Whybrow S, Mayer C, Kirk TR, Mazlan N, Stubbs RJ (2007) Effects of two weeks' mandatory snack consumption on energy intake and energy balance. Obesity (Silver Spring) Mar; 15(3), 673-85.

3. Astbury N, Taylor M, Macdonald I (2008) The effects of a polydextrose preload on appetite and energy intake. P Nutr Soc 67, E342. 